\title{
Control of the objects with a single output and with two or more input channels of influence
}

\author{
V. A. Zhmud ${ }^{1}$, O. D. Yadrishnikov ${ }^{1}$ \& V. M. Semibalamut ${ }^{2}$ \\ ${ }^{I}$ Novosibirsk State Technical University, Russia \\ ${ }^{2}$ Geophysical Service of SB of Russian Academy of Science, Russia
}

\begin{abstract}
The control of dynamic objects inside the locked loop with negative feedback is now widely used in automation and robotics. This ensures greater precision under the action of uncontrolled disturbances. Usually, for controlling the single output value of the object only one input action on the object is sufficient. But there are cases where it is advisable to apply two or more feedback control actions to the object for a single output value controlling. Such a case occurs when the structure of the mathematical model of the object allow the influence to the output value in different ways, every of which corresponds to different mathematical models. Each of these models has their own speed limits on the maximum value of the actions of limited frequency band, and so on. Joint use of the multiple control channels can simultaneously provide both high speed control and a large range of controlled changes in the output value of the object. This requires the use of multiple regulators, jointly acting on the object. Coordination of dynamic and static properties of these regulators has been discussed theoretically using transfer functions. But it does not take into account such a limitation of the control channels of the object as a dynamic non-linearity. This article discusses these limitations one of the channels. It uses mathematical modeling for transient processes and to optimize the regulators. Advantages of using this method are investigated by examples. It is for the first time in this paper that the mathematical modeling and numerical optimization for finding the best values of the coefficients of the various regulators in such a structure were used. The simulation showed the advantages of this method and its limitations for use. The paper is illustrated with the graphs obtained transient processes in the locked system.
\end{abstract}

Keywords: control, feedback, automation, regulator, quality of control, accuracy. 


\section{Introduction}

Control of objects with negative feedback loop is widely used because it allows achieving high accuracy in the presence of disturbances. In such systems, the control error $\mathrm{e}(\mathrm{t})$, equal to the difference between the output signal of the prescribed object output value $\mathrm{v}(\mathrm{t})$ and the real output value $\mathrm{y}(\mathrm{t})$, are converted into a control signal $\mathrm{u}(\mathrm{t})$, which is applied to the object input. The result of this is to achieving of the desired approximate equation of the two signals $y(t) \approx v(t)$, at least on average, (inside a limited frequency bandwidth). In some objects there are several output values, and there are several channels of influence on these output values. Typically, the number of inputs controlling channels in the object corresponds to the number of controlled output variables. If the number of input channels is less, some of the output values must left to be unmanageable. If the number of input channels is more than the number of output values, then they usually choose the most effective controlling channels and excess channels are not used.

In some cases it is advisable to use more control channels than number of output values in the object. The examples of such control system are the system for the control of frequency or phase of the laser light [1-3]. In such systems, the structure of the object allows controlling of the output value in different ways, which correspond to different mathematical models of the corresponding channels. With all this each of these models has their own speed limits on the maximum value of the impact and so on. Jointed control with the help of the both channels can simultaneously provide both high speed control and a large range of controlled changes of the output value of the object. This requires the use of two regulators jointly acting on the object.

Problem of calculation of dynamic and static properties of these regulators were considered theoretically using transfer functions [4-14], however, these papers have not considered such restrictions of one of the control channels of the object as a dynamic non-linearity. This paper discusses these limitations of one of the channels, using the mathematical modeling for calculation of the transient processes and to optimize the regulators. Features of using of this method are investigated by an example with modelling.

\section{Statement of the problem}

Let's consider an object, the output value of which can be controlled using two different channels, one of which has poor speed, but sufficient range of changes, and the other one, on the contrary, has big range of variation of the output value, but poor speed, which is not enough [4]. For example, in the control phase or frequency of the laser radiation [1-3] for measuring of the frequency (or phase) the device uses the changing the optical length of the laser, which is determined by the distance between the two mirrors. Any one or both of these mirrors may be arranged on piezoelectric modulator which changes its dimensions (to a small amount) under the influence of the voltage applied to it. If using one modulator 
the smallest size, it can provide high performance, but can only alter the radiation frequency by a small amount. If we use the gluing of several of modulators, we can provide the change in frequency by a sufficiently large desired value, but such bonding has a much lower speed, which affects in limiting of the rate of rise of the output variable. This limited performance can be described as limited value of the slew rate of the output variable (dynamic nonlinearity).

Thus, one channel can control the output value rapidly, but in a small range (fast channel) the other channel can act on it over a large range, but with limited speed (slow channel).

Properties of the laser frequency noise are similar to the jointed possibilities of the control channels. Namely, largest laser frequency changes are slow, and rapid changes in frequency are small.

It follows from this that the combination of two feedback channels might allow to suppress both types of perturbations in the system. However, it is necessary to avoid the conflict between the two types of controls of the only output value.

The paper [4] has proposed a theoretical analysis of the problem and its solution based on the logarithmic amplitude-frequency characteristics. But the object model is often so complicated that the analytical methods are not effective for solving of the stated problem. Furthermore, these kinds of limitations in the fast and slow channel (level and slew rate of the output signal) are difficult to take into account in the analytical assay, whereas the optimization using the simulation program with these limits can be easily done.

Thus, it is advisable to consider models that take into account these limitations and to offer adequate models for them. These models are used for numerical optimization of the regulator. Then we can compare the results with the processes which can be obtained the use of only any one of the channels. All simulation is done in the program VisSim 6.0.

\section{The model of the slow channel}

To describe the slow channel we can be use first-order link with the introduction of limited slew rate into it. Without this restriction said link can be modeled as a circuit with negative feedback which contains an integrator in the forward channel. Such structure produces at the output of the integrator an output value of this model, and at its input the time derivative of this value is calculated, which is the slew rate of the output value. If we introduce limiter before the integrator, then the resulting model will describe a device that with small signals is described as first-order, but for large input signals it has limitation to the speed of increase of the output value.

An example of such a channel simulation is shown in fig. 1. The amplitude of the signal at the output of such a model depends on the frequency and amplitude of the input signal; it is nonlinear relatively of the slew rate. 


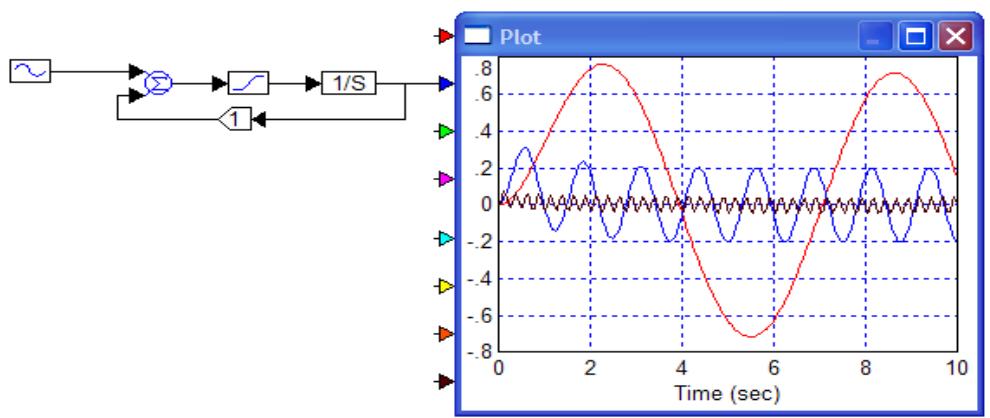

Figure 1: The model of the slow channel: the limiter has thresholds \pm 1 ; oscillator output amplitude is 1 , frequency is $1 \mathrm{~Hz}$ for the biggest line, $5 \mathrm{~Hz}$ for the middle-value line and $25 \mathrm{~Hz}$ for the smallest line; feedback loop is the model of the dynamic non-linear link.

Figs 2 and 3 show the output of the model with different ratios of the frequency and amplitude of the input signal. At a fixed frequency with increasing output signal amplitude first increases, but after a certain value it becomes of saw tooth shape, and the amplitude stops its growth. The shown model is adequate to the slow channel of control of the laser light frequency [4].

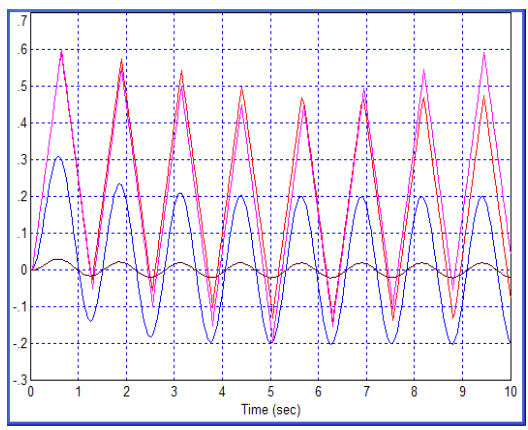

Figure 2: Dynamic non-linearity of the model with the growth of the amplitude when the frequency is $1 \mathrm{~Hz}$.

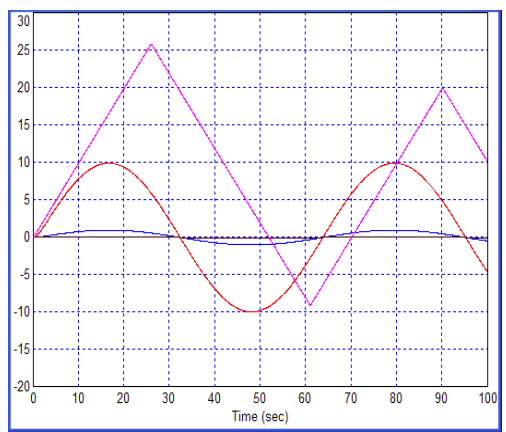

Figure 3: Dynamic non-linearity of the model with the growth of the amplitude when the frequency is $0.1 \mathrm{~Hz}$.

\section{The model of the fast channel}

To describe the fast channel it is advisable to use first-order link with limiter unit at its input. Fig. 4 shows a model of fast channel. It includes limiting the magnitude of \pm 2 and first-order link with a time constant of $1 \mathrm{~s}$. With the growth of the amplitude of the output signal, its form becomes transformed from the harmonic one into a limited signal shaped like a trapezoidal one. 


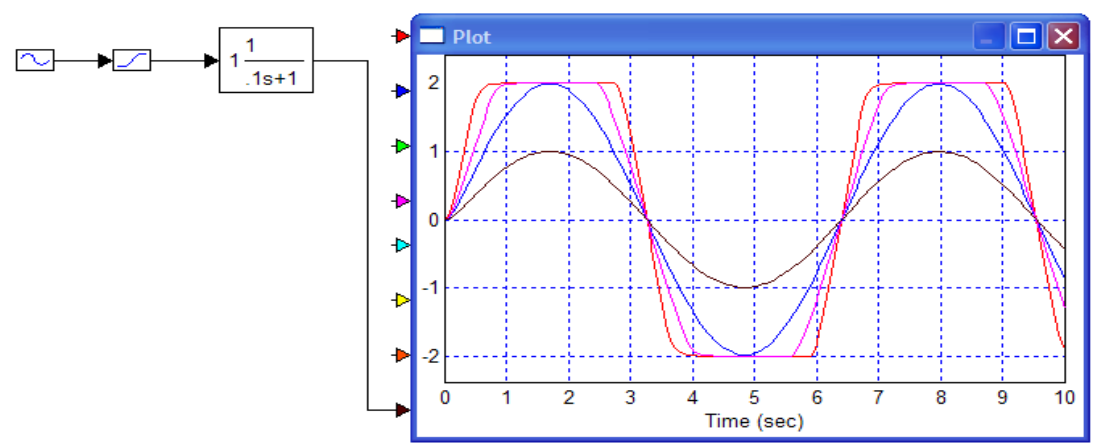

Figure 4: The model of fast channel and its output signals with various amplitude of the input signals: 1, 2, 3 and 5 units.

\section{The model of the object as a whole and model of the disturbance}

Fig. 5 shows an object model that combines both the control channel. Oscilloscope, which is the part of the modeling program VisSim, shows that with increasing of amplitude the output signal has harmonic distortion, which is the sum of signals corrupted by each of the control channels. Of course, the inputs of the both channels are not required to be connected. On the contrary, each channel must be feed by its own control signal, so it is necessary to calculate the twochannel regulator.

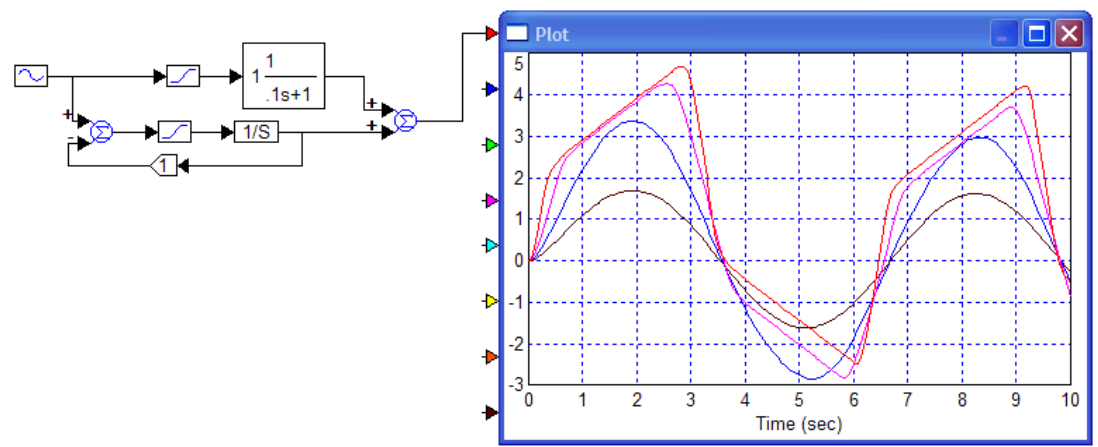

Figure 5: Joined object model and its output signals with the changing amplitude of the input signal: 1, 2, 4 and 8 units.

All uncontrolled processes in the object can be described as disturbance acting on the output and summed with the output signal, which is the response generated by the two control channels to the signals on their inputs. Let disturbance component has amplitude of 20 units at a frequency of $0.02 \mathrm{~Hz}$ and an additive component with the amplitude of 1 unit at a frequency of $1 \mathrm{~Hz}$. Fig. 6 shows an example of such disturbance. 


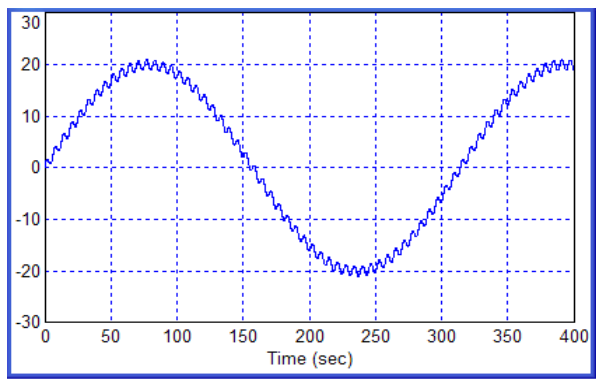

Figure 6: Typical view of the disturbance as a sum of the two components.

The system was unable to suppress such interference only with fast channel or only with the slow channel. The goal is to suppress this disturbance with the joint action of the two channels. For this purpose it is necessary to select the structure of the regulator and calculate the coefficients for it.

\section{Solving of the problem by numerical optimization}

The regulator must have integrator in the slow channel and derivation on the fast channel. It is not so clearly to determine which of the two channels should include the proportional link. To simplify the preliminary analysis and in the order to ensure that all the possibilities are fully included to the regulator model, we propose the use of the proportional in the both of them. If the numerical optimization resulting coefficient of one of these channels will be negligible for the proportional link, then this link will be excluded from the final version of the regulator.

Numerical optimization techniques are studied in [5-14]. For the reasons stated in [4], we recommend using program is VisSim, rather than MATLAB or MathCAD.

Fig. 7 shows a model for the numerical optimization of the regulator. Fig. 8 shows the resulting transition process, which is the remainder of the disturbance. It is suppressed by the control loop. It is seen that the interference amplitude which is 20 units as a whole is suppressed to a value not greater than 0.15 units, i.e. more than 100 times.

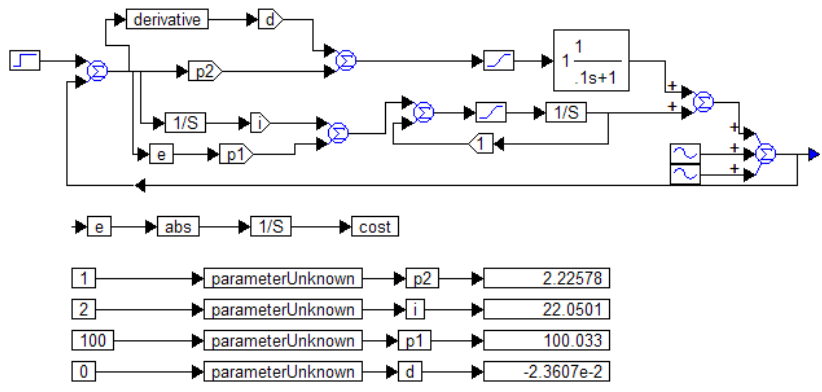

Figure 7: Structure for optimization in VisSim. 


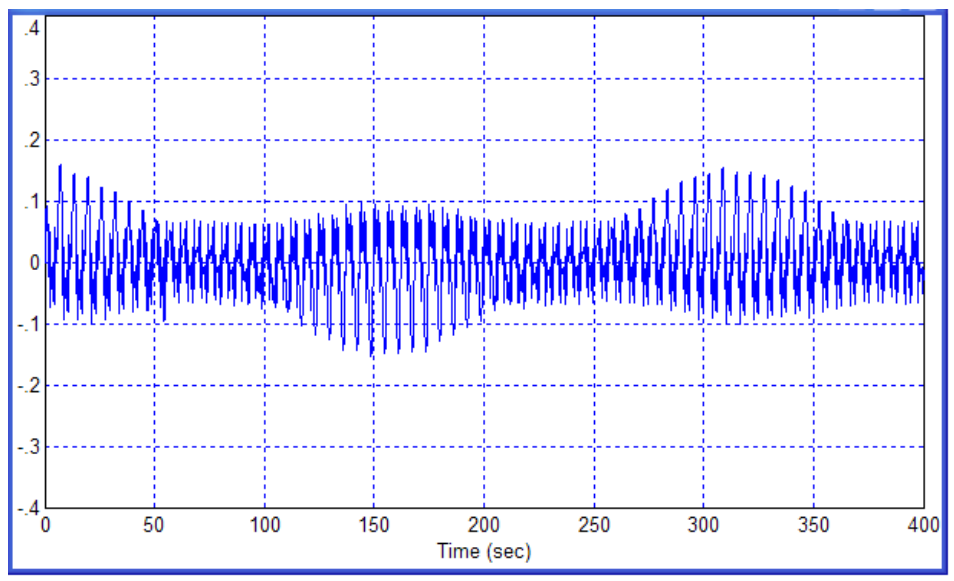

Figure 8: The view of the reminders of the disturbance by the feedback loop.

\section{Methods and results of the further improvement of the system}

We should try to improve the result of optimization, because it is clear that the derivative channel does not work. It follows from these two indicators: the relatively small value of the coefficient obtained for this link and its negative value. Derivative link usually improves phase stability reserve, which increases system performance. The gain of such link tends to be positive. In the object there is no lag, so derivation should be usable. It is necessary to find out the reason of what is happening and try to remove it. To begin, we can offer to fix all the coefficients except derivative link, and then we apply a single step jump to the input. We also remove high-frequency component of the disturbance. As a result of the optimization procedure is the obtained gain of the derivative link $K_{\mathrm{D}}$ $=-0.09$.

Since the said ratio remained negative and the amplitude balance interference not only did not fall, it should be recognized that the done manipulation did not improve the result. Therefore, we should consider in detail the signals in the system, and on the basis of this review, to draw attention to the fact that the limiter after derivative device negates the effect of derivation, transforming it from the useful properties of harmful. It is the combination of derivation with subsequent restriction is the main source of problems with derivation. If not for that, the majority of practitioners and theorists who fear of the derivation of the noisy signals did not meet to confirm for their fears. We note that the proportional path also acts on a limiter, which gives a negative effect. To solve this problem, proceed as follows. We define the gain of the derivative link in the fast regulator as a small positive value, namely 0.005 . We also disable slow big disturbance and slow regulator and accomplish near optimization only for the gain of the derivative link. The structure of such a procedure is shown in fig. 9 . 


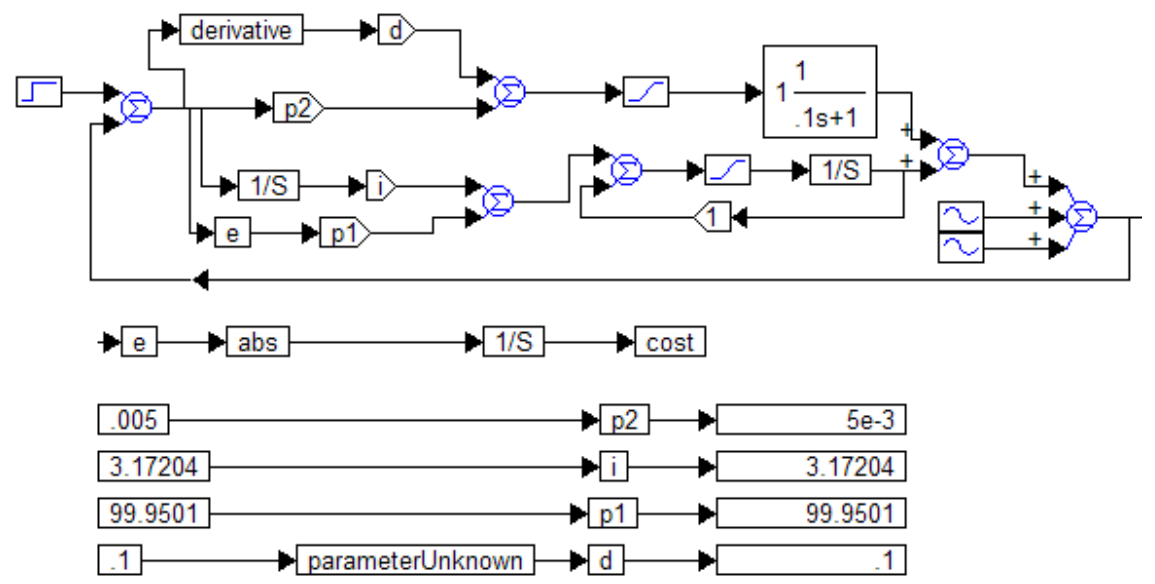

Figure 9: The structure of the further optimization for derivative link.

As a result of optimization carried out by the structure shown in fig. 9, the derivation gain has a positive value $K_{\mathrm{D}}=0.1$. Fig. 10 shows remains of disturbance in the result of the working of the final regulator. One can see that the residual error at the output remains within about \pm 0.1 , and the derivative link has a positive effect.

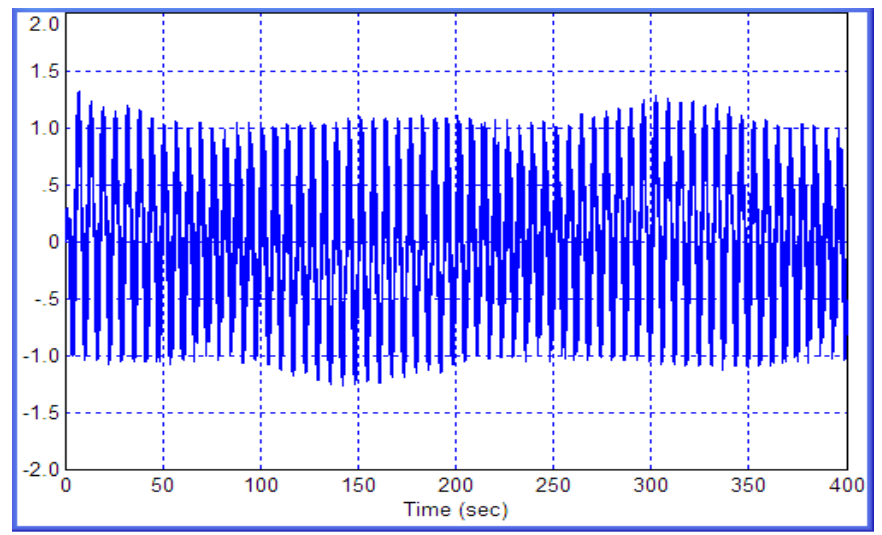

Figure 10: The remains of the disturbance in the final system.

\section{Another example with a lower limitation level}

In order to ensure that the cause of the problems was namely in the limitation of the signal, let increase the threshold limit on the value from \pm 2 to \pm 5 units, that is only 2.5 times. The structure for the optimization and the results of it are shown in fig. 11. As a result, the derivative link gain becomes a large positive value, as follows: $K_{\mathrm{D}}=14.36$. 


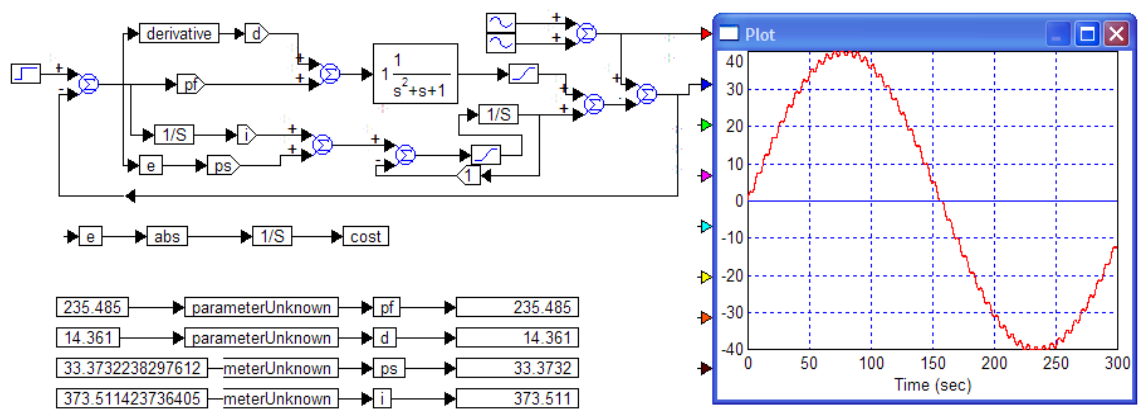

Figure 11: The results of the optimization after the increasing of the level of limitation.

Fig. 12 shows the remains of the disturbance when the output signal is the same as earlier. Input reference signal $\mathrm{v}(\mathrm{t})$ in this case is equal to zero, so this output signal, in fact, is an error of the control.

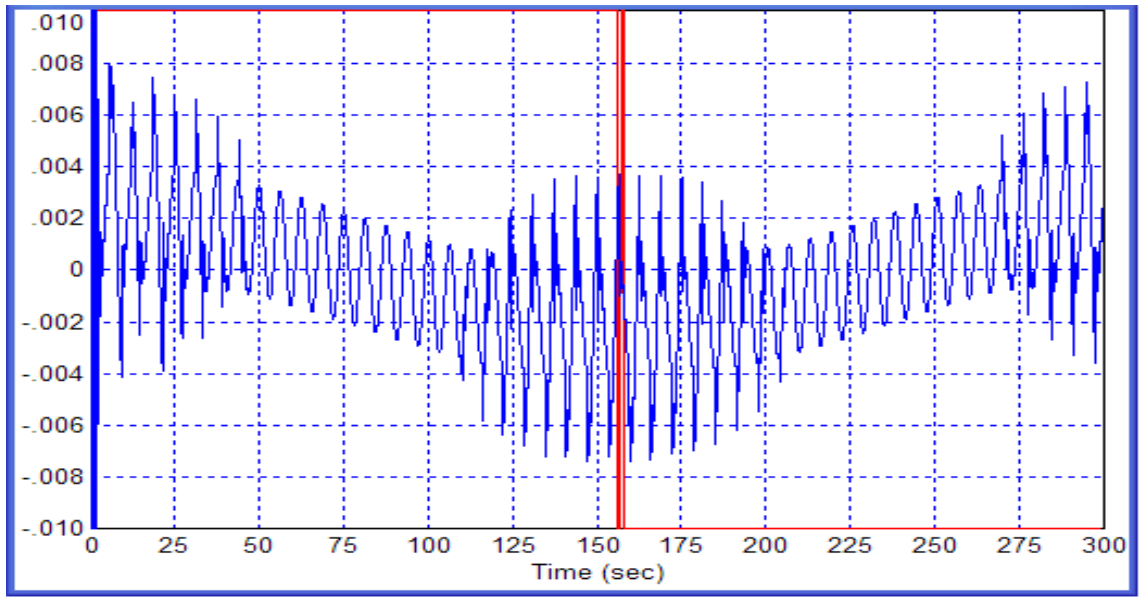

Figure 12: The residual error of the system shown at fig. 11.

The output signal amplitude only in some cases reaches a value of 0.008 , and sometimes it is 0.002 . Bursts of high-frequency noise generated at the moments when the disturbance approaches zero, so it is possible to assume that a small conflict of the two control regulators still takes place. Overcoming of this conflict probably would allow further reduce of the amplitude of the residual noise. However, the result is completely satisfactory: the interference is suppressed, at least 2500 times. 


\section{The demonstration of the effectiveness of the use of two control channels}

To demonstrate the effectiveness of the investigated method is sufficient to consider the result without any of the channels. Fig. 13 shows the output of the system, which is the error generated by disturbance when suppressed by the single regulator loop, when the fast channel is disconnected. Fig. 14 shows the output of the system when the slow control channel is disconnected. In every case, the residual regulator was preliminary numerically optimized with the program VisSim.

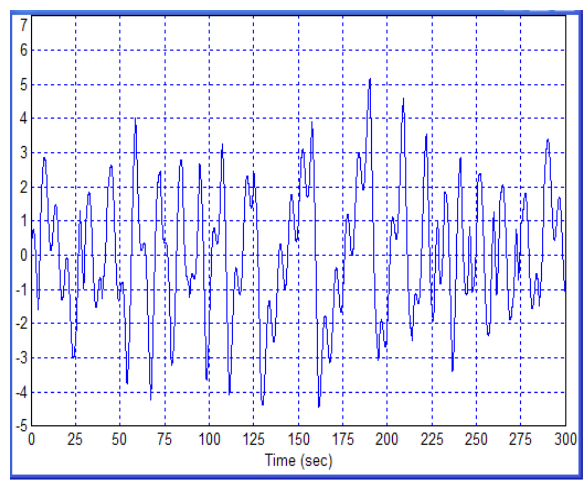

Figure 13: The residual error in the system with the slow regulator only.

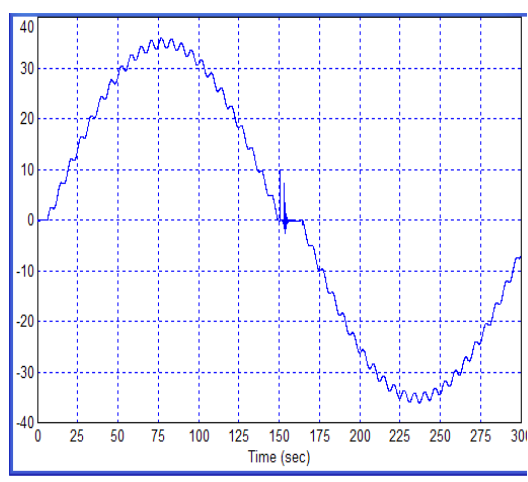

Figure 14: The residual error in the system with the fast regulator only.

\section{The indicators of the effectiveness of each channel}

It is advisable to make sure that there are no signs of inefficiency of each channel. An indication of poor performance in fast signal channel would limit signal therein or the presence of large DC offset. Limitation would indicate congestion channel, and displacement would reveal not enough for efficient operation of the slow channel. Fig. 15 illustrates fast channel output, it is seen that these attributes are not contained therein.

An indication of poor performance in the signal of the slow channel would residues of the high-frequency signal, particularly if it were present in the nonlinear distortion, which would transform harmonic signal into a saw-tooth shape.

Fig. 16 shows that the output signal of slow channel signal is good. Thus, we can conclude that as a result of numerical optimization the complex regulator is designed which provides efficient separation of modes of transient process for two separated regulator of the common single controlling output value. 


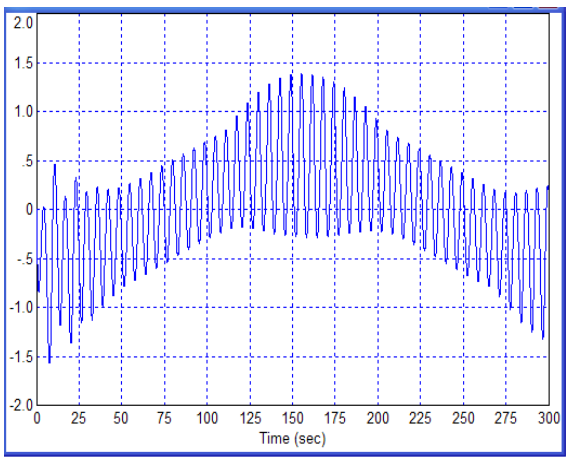

Figure 15: The output signal of the fact regulator.

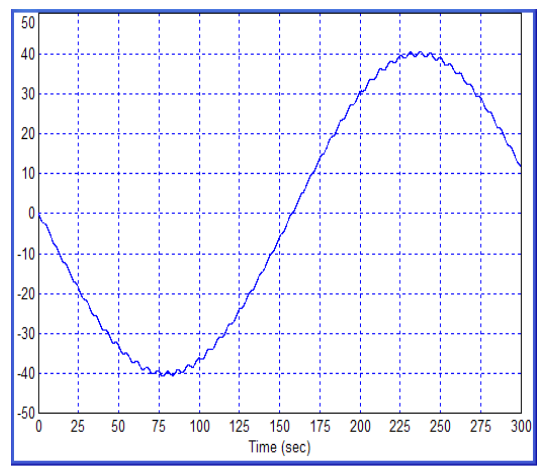

Figure 16: The output signal of the slow regulator.

\section{Conclusion}

The paper has studied the method of calculating of the two-channel regulator for the only output variable based on numerical optimization. The causes of not effective work of the fast channel is revealed, it is the lack of accounting of the limitations, which leads to the fact that the differentiation instead of being the useful properties becomes harmful one, so the corresponding coefficient is negative. The paper has given the recommendations to overcome the discussed problem.

\section{Acknowledgement}

This work was financially supported by the Russian Ministry of Science and Education, State job №2014/138, the theme of the project is "New structures, models and algorithms for the control of breakthrough technology systems based on high-tech results of intellectual activity".

\section{References}

[1] A phase-locked loop system for the difference frequency of two lasers. Barmasov S.V., Zhmud' V.A. Instruments and Experimental Techniques. 2000. T. 43. № 3. pp. 381-383.

[2] An electronic system for stabilizing of the frequency of the He-Ne laser to the methane absorption lines. Zhmud V.A., Barmasov S.V., Gitel'son V.D. Instruments and Experimental Techniques. 1999. T. 42. № 4. pp. 551-557.

[3] V. A. Zhmud. The Use of the Feedback Control Systems in Laser Physics Researching Experiments. // Proceedings of RFBR and DST Sponsored "The 2nd Russian-Indian Joint Workshop on Computational Intelligence and Modern Heuristics in Automation and Robotics", 10-13 September, 2011, Additional volume, pp. 40-43. 
[4] V.A. Zhmud. Simulation, research and optimization of locked system of automatic control. Monograph. Novosibirsk. Publishing house of NSTU. 2012, p. 335.

[5] V.A. Vasiliev, A.A. Voevoda, VA Zhmud, V.A. Hassuoneh. Digital controls: cost function, the choice of integration method, hardware implementation. Collection of scientific works of NSTU, 2006. N 4 (46). pp. 3-10.

[6] A.S. Vostrikov, A.A. Voivod, V.A. Zhmud. The effect of reducing the order of the system that is controlled by the method of separation of motions. Scientific Bulletin of the NSTU. - 2005. - N 3 (21). pp. 3-21.

[7] A.A. Voevoda, V.A. Zhmud. Maintenance and improvement of the order of the asymptotic equation of the system that is controlled by the method of separation of motions. Scientific Bulletin of the NSTU. - 2006. - N 1 (22). pp. 3-9.

[8] A.A. Voevoda, V.A. Zhmud. The convergence of the optimization algorithms for object control with limiter and delay. Scientific Bulletin of the NSTU. - 2007. - N 4 (29). pp. 179-184.

[9] The modeling tests of the new PID-regulators structures. Voevoda, A.A., Zhmud, V.A., Ishimtsev, R.Y., Semibalamut, V.M. 2009. Proceedings of the IASTED International Conference on Applied Simulation and Modelling, ASM 2009. pp. 165-168.

[10] Modern key technologies in automatics: Structures and numerical optimization of regulators. Zhmud, V., Yadrishnikov, O., Poloshchuk, A., Zavorin, A. 2012. Proceedings - 2012 7th International Forum on Strategic Technology, IFOST 2012.

[11] The design of the feedback systems by means of the modeling and optimization in the program vissim 5.0/6. Zhmud, V., Liapidevskiy, A., Prokhorenko, E. 2010. Proceedings of the IASTED International Conference on Modelling, Identification and Control. pp. 27-32.

[12] V. Zhmud, O. Yadrishnikov. Numerical optimization of PID-regulators using the improper moving detector in cost function. Proceedings of the 8th International Forum on Strategic Technology 2013 (IFOST-2013), vol. II, 28 June-1 July. Mongolian University of Science and Technology, Ulaanbaator, Mongolia. IEEE organized. 2013. pp. 265-270. http://www.must.edu.mn/IFOST2013/.

[13] V. Zhmud, A. Polishchuk, A. Voevoda, R. V. Rao. The Tuning of the PID-Regulator for Automatic Control System of Thermo Energetic Equipment // Proceedings of the Fifth International Conference on Advances in Mechanical Engineering (ICAME-2011), June 06-08, 2011. Surat - 395 007, Gujarat, India. pp. 254-263.

[14] Zhmud V.A., Zavorin A.N. Metodi di ottimizzazione del controllo numerico su una modelli troncati. Italian Science Review. 2014; 4(13). pp. 686-689. Available at URL: http://www.ias-journal.org/archive/2014/ april/Zhmud.pdf and http://www.ias-journal.org/archives/april-2014. 